\title{
A NOTE ON THE LIE ALGEBRAS OF ALGEBRAIC GROUPS
}

\author{
TSUneo KanNo
}

(Received July 2,1958)

0. In his book |1] C. Chevalley defined the replicas for any elements of Lie algebras of algebraic groups of matrices which are defined over fields of characteristic 0 , and he characterized algebraic subalgebras as those subalgebras of the general linear algebras which are closed with respect to "replica operation" i.e. those which contain all replicas of any elements of themselves. In this paper we shall define the replica in the case of any algebraic groups defined over fields of characterestic 0 and show that the same characterization of algebraic subalgebras is true in this case too.

1. Let $G$ be a connected algebraic group ${ }^{1)}$; let $\Omega(G)$ be the field of rational functions on $G$ where $\Omega$ is the universal domain; let $k(G)$ be the subfield of $\Omega(G)$ consisting of all rational functions defined over $k$ where $k$ is a field of definition for $G$. Then $\Omega(G)$ is the union of $k(G)$ for all fields $k$ of definition for $G$, and the mapping $f \rightarrow f(p)$ is a $k$-isomorphism of $k(G)$ onto $k^{\prime}(p)$ where $p$ is a generic point over $k$ on $G$. Suppose that $G$ is given by $\left[V_{\alpha}, \mathfrak{\mho}_{\alpha}, T_{\beta \alpha}\right]$, and let $\xi_{1}, \ldots, \xi_{N}$ be the coordinate functions relative to $V_{\alpha}$, i.e. $\xi_{i}(p)=x_{i}$, where $(x)$ is the representative of $p$ in $V_{\alpha}$. Then we have $\Omega(G)=\Omega(\xi)$ and $k(G)=k(\xi)$.

For any $p \in G$ we denote by $\mathfrak{o}_{p}$ the local ring of $p$ on $G$. Let $\mathfrak{m}_{p}$ be the maximal ideal of $\mathfrak{o}_{p}$. By a tangent vector to $G$ at $p$ we mean an $\Omega$-linear mapping $X_{p}$ of $\mathfrak{o}_{p}$ into $\Omega$ such that for $f_{1}, f_{2} \in \mathfrak{o}_{1}$, we have

$$
X_{p}\left(f_{1} f_{2}\right)=\left(X_{p} f_{1}\right) f_{2}(p)+f_{1}(p)\left(X_{p} f_{2}\right) .
$$

If $k$ is a field of definition for $G$ such that $p$ is rational over $k$, then $X_{p}$ is said to be rational over $k$ if $X_{p}$ maps $\mathfrak{o}_{p} \cap k(G)$ into $k$. An $\Omega$-derivation $D$ is said to be finite at $p$ if $D$ maps $\mathfrak{o}_{p}$ into itself. In this case $D$ induces a tangent vector $D_{p}$ to $G$ at $p$ such that $D_{p} f=(D f)(p)$ for $f \in \mathfrak{o}_{p}$, which is called the local component of $D$ at $p$. If further $D$ is defined over $k$ and maps $\mathrm{m}_{p} \cap k(G)$ into itself, we have a $k$-derivation $X$ of $k(p)$ such that $X f(p)$ $=\left(D f^{\prime}\right)(p)$ for $f(p) \in k^{\prime}(p)$, where $f^{\prime}$ is an element of $\mathfrak{o}_{p} \cap k(G)$ such that $f^{\prime}(p)$ $=f(p)$.

Let $\rho$ be a rational mapping of $G$ into another connected algebraic group $G^{\prime}$. If $\rho$ is generically surjective, we get an $\Omega$-isomorphism $\rho^{*}$ of $\Omega\left(G^{\prime}\right)$ into $\Omega(G)$ such that $\rho^{*} f(x)=f(\rho(x))$ for $f \in \Omega\left(G^{\prime}\right)$, where $x$ is a generic point on $G$ over some field of definition for $G, G^{\prime}, \rho$, and $f$. For $p \in G$ let $R_{p}$ be the right translation, let $L_{p}$ be the left translation, and let $\iota(p)$ be the inner

1) As for the terminology and preliminary results, cf. Nakano [2] an 1 Rosenlicht $[3],[4]$. 
automorphism $x \rightarrow p x p^{-1}$ of $G$. Any $\Omega$-derivation $D$ of $\Omega(G)$ is called (right) invariant if $R_{p}^{*} \quad D f=D R_{p}^{*} f$ for any $p \in G$ and $f \in \Omega(G)$. Any invariant $\Omega$ derivation is everywhere finite and determined by the local component at one point on $G$. The set of all invariant $\Omega$-derivations of $\Omega(G)$ is called the Lie algebra of $G$ which is a Lie algebra over $\Omega$ with the bracket multiplication $[D, D]=D D^{\prime}-D^{\prime} D$. In the case of any algebraic group, the Lie algebra of its component containing the unit element $e$ is called the Lie algebra of this algebraic group. In this paper we shall denote algebraic groups by $G, G^{\prime}, H, \ldots$, and their Lie algebras by $\mathfrak{g}, \mathfrak{g}^{\prime}, \mathfrak{h}, \ldots$. It is known that if $K$ is a field of definition for $G$, the Lie algebra $g$ of $G$ has a base consisting of $n$ invariant $\Omega$-derivations defined over $K$, where $n$ is the dimension of $G$. The set $g(, K)$ of all those elements of $g$ which are defined over $K$ is a Lie algebra over $K$, and $\mathfrak{g}$ is the scalar extension of $\mathfrak{g}(, K)$ from $K$ to $\Omega$.

Let $\rho$ be a rational homomorphism of $G$ into $G^{\prime}$, then we have a homomorphism $d \rho$ of $\mathfrak{g}$ into $\mathfrak{g}^{\prime}$ such that $\rho^{*}(d \rho(D) f)(p)=\left(D \rho^{*} f\right)(p)$ for $D \in \mathfrak{g}, \quad p \in G$ and $f \in 0_{\rho p}$. Let $H$ be a connected algebraic subgroup of $G$, and let $\sigma$ be the natural injection of $H$ int $G$, then $d \sigma(\mathfrak{h})$ is a subalgebra of $g$ which is $\Omega$ isomorphic to $\mathfrak{h}$. In this paper we identify $d \sigma(\mathfrak{y})$ with $\mathfrak{y}$. Then an element $D$ of $\mathfrak{g}$ is in $\mathfrak{y}$ if and only if $D$ maps $\mathfrak{m} \cap k(G)$ into itself, where $k$ is a field of definition for $G, H$ and $D$ and $m$ is the maximal ideal of the local ring of a generic point over $k$ on $H$. A subalgebra of $g$ is called algebraic if it is the Lie algebra of some connected algebraic subgroup of $G$.

Let $k$ be a field of definition for $G$; let $x$ and $y$ be independent generic points over $k$ on $G$; let $\phi$ be the rational mapping of $V_{\alpha} \times V_{\alpha}$ into $V_{\alpha}$ which is induced by the group operation $G \times G \ni x \times y \rightarrow x y \in G$; let $\phi^{z}(x, y)$ be the $i$-th coordinate of the representative of $x y$ in $V_{a}$; let $\varphi^{i}(X, Y)$ be a suitable rational expression in indeterminates $(X ; Y)$ with coefficients in $k$ (e.g. if the unit element $e$ has a representative in $V_{a}$ we take such $\phi^{i}(X, Y)=P^{i}(X$, $Y) / Q^{2}(X, Y)$ that $Q^{i}(e, e) \neq 0$, where $P^{i}, Q^{\prime} \in k[X, Y]$. For any $\Omega$-derivation $D$ of $\Omega(G)$, put $D \xi_{i}=\chi_{i}(\xi)$. Then $D$ is determined by $\left(\chi_{1}(\xi), \ldots, \chi_{N}(\xi)\right)$. If a point $z$ of $G$ has a representative in $V_{\alpha}$ and $D$ is finite at $z$, the local component of $D$ at $z$ is determined by $\left(\chi_{1}(z), \ldots, \chi_{N}(z)\right)$. If $D$ is defined over $k, \chi_{i}(\xi)$ is in $k(\xi)$. And $D$ is invariant if and only if

$$
\chi_{i}\left(R_{y}^{* \xi}\right)=\sum_{j=1}^{v}\left(\partial \mathcal{P}^{i}(X, y) / \partial X_{j}\right)_{X=\xi} \chi_{j}(\xi) .
$$

If the unit element $e$ has a representative in $V_{\alpha}$, we have

$$
\chi_{i}(y)=\sum_{j=1}^{N}\left(\partial \varphi^{i}(X, y) / \partial X_{j}\right)_{\Gamma=e} \chi_{j}(e),
$$

and therefore

$$
\chi_{i}(\xi)=\sum_{j=1}^{v}\left(\partial \phi^{i}(X, \xi) / \partial X_{j}\right)_{X=e} \chi_{j}(e) .
$$

Conversely if this relation holds for an $\Omega$-derivation $D$ defined over $k$ such that $D \xi_{i}=\chi_{i}(\xi)$, then $D$ is invariant.

In the following we often denote by the same letter $x$ the point of $G$ and 
its representative in some affine variety $V$.

Suppose that $G$ is a connected algebraic subgroup of $\left.G L^{\prime} n, \Omega\right)$. Let $u_{i}$, and $\xi_{i j}$ be the coordinate functions of $G L^{\prime} n, \Omega$ ) and $G$, respectively; let $D$ be an element of $\mathfrak{g}$ defined over $k$; let $\mathfrak{p}$ be the prine ideal of $k[u]$ associated with $G$; put $D \xi_{i j}=\chi_{i j}(\xi)$, then (2) implies that

$$
D \xi_{, j}=\sum_{i=1}^{\mathrm{v}} \chi_{l l}(I) \xi_{l j}
$$

where $I$ is the unit matrix. Put

$$
\Phi(D)=-\left(\chi_{i j}(I)\right),
$$

then the $k$-derivation $\delta(\Phi(D))$ of $k[u]$ maps $p$ into itself ${ }^{2)}$. A simple calculation shows that $D \rightarrow \Phi(D)$ is a $k$-isomorphism of $\mathfrak{g}(, k)$ into $\mathfrak{g l}(n, k)$ and that image of $\mathfrak{g}(, k)$ by $\Phi$ is the Lie algebra of $G$ defined by Chevalley [1] p. 128. Thus we may imbed the Lie algebra of algebraic subgroup of $G L(n, \Omega)$ in $\mathfrak{l}(n, \Omega)$.

The next le.nma is usefull in the section 3 .

Lemma 1. Let $s$ be a point on $G$; let $k$ be a field of definition for $G$. Then for a k-derivation $X$ of $k(s)$ there exists uniquely an element $D$ of $\mathfrak{g}$, defined

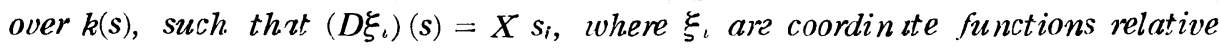
to $V$ in which s has a representative.

Proof. If we set $X_{s} f=(X f(s))$ for $f \in 0_{s} \cap k(G)$, weobtain a $k$-linear mapping $X$; of $\left.\mathfrak{o}_{s} \cap k^{\prime} G\right)$ into $k(s)$ such that for $f_{1}, f_{2} \in \mathfrak{v}_{s} \cap k(G)$

$$
X_{s}\left(f_{1} f_{2}\right)=\left(X f_{1}\right) f_{2}(s)+f_{1}(s)\left(X_{*} f_{2}\right)
$$

Let $K$ be any overfield of $k(s)$. Then for any $f \in K[\xi]$ we may express $f=$ $\sum_{i=1}^{n} \alpha f_{i}$, where $\alpha_{i} \in K$ and $f_{i} \in k[\xi]$. If we set $X_{s} \bar{f}=\sum_{i=1}^{n} \alpha_{i} X_{\odot} f_{i}$, we obtain a $K$-linear mapping $X_{s}$ of $K[\xi]$ into $K$ with the analogous property (4) for $f_{1}, f_{2} \in K[\xi]$. In fact; suppose that $\sum_{i=1}^{n} \alpha_{i} f_{i}=0$. Then we may suppose that for some intger $l \leqq m \alpha_{1}, \ldots, \alpha_{l}$ a re linearly independent over $k$ and $\alpha_{i}=\sum_{j=1}^{l} \gamma_{i j} \alpha_{j}$ for some $\gamma_{i j} \in k$. The equation $\sum_{l=1}^{l} \alpha\left(f_{i}+\sum_{l=l+1}^{m}\right.$ $\left.\gamma_{j} f_{j}\right)=0$ implies $f_{i}+\sum_{j=l+1}^{n} \gamma_{j} f,=0$, since $K$ and $k(\xi)$ are linearly disjoint over $k$. Thus we have $X_{s} f_{i}+\sum_{i=l+1}^{n} \gamma_{j i} X_{s} f_{j}=0$ and $\sum_{i=1}^{n} \alpha_{i} X_{s} f_{i}=\sum_{i=1}^{l} \alpha_{i}\left(X_{s} f_{i}\right.$ $\left.+\sum_{j=l+1}^{n} \gamma_{i i} X_{s} f_{j}\right)=0$, and the mapping of $K[\xi]$ into $K$ is defined. The linearity is clear and the equation (4) holds for such forms $\alpha_{1} f_{1} . \alpha_{2} f_{2}$ that $\alpha_{1}, \alpha_{2} \in K$ and $f_{1}, f_{2} \in k[\xi]$, Clearly $X_{s}$ induces, a tangent vector to $G$ at $s$ which we shall denote by the same $X_{s}$. Taking $K=k^{\prime} s$ ), we see that $X_{s}$ is rational over $k(s)$.

Let $f$ be an element of $\Omega(G)$; let $K$ be a field of definition for $G$ and $f$ over which $s$ and $X_{s}$ are rational let $x$ be a generic point on $G$ over $K$. Then

2) As for the definition of $\delta$ cf. [1] p. 126 
$R_{s}^{*}-1_{\gamma} f$ is in $0_{s}$ and rational over $K(x)$, so $X_{s} R_{s-1,}^{*} f$ is a welldefined element of $K(x)$. Let $f^{\prime}$ be the unique element of $k(G)$ such that $f^{\prime}(x)=X_{s} R_{s-1}^{*} f$. It is clear that $f^{\prime}$ depends only on $G, f$ and $X_{s}$. If we set $D f=f^{\prime}$, we obtain the element $D$ of $g$ described above. In fact, $D \Omega=0$ and the linearity holds. For $f_{1}, f_{2} \in K(G)\left(D, f f_{1}\right)(x)=X_{s} R_{s-1_{x}}^{*}\left(f_{1} f_{2}\right)=X_{s}\left(R_{s-1_{x}}^{*} f_{1} \quad R_{s-1}^{* 1} f_{1}\right)=\left(D f_{1}\right)(x) f_{2}(x)$ $+f_{1}(x)\left(D f_{2}\right)(x)$. Taking $\left.K=k^{\prime} s\right)$, we see that $D$ is an $\Omega$-derivation of $\Omega(G)$ defined over $k(s)$. If $f \in \Omega(G), a \in G, K$ is a field of definition for $G$ and $f$ over which $a, s$ and $X_{s}$ are rational, and $x$ is generic for $G$ over $K$, we have $\left(R_{t}^{*} D f\right)(x)=\left(D f ;(x a)=X_{s} R_{s-1}^{*} x a=X_{s} R_{s-1_{x}}^{*} R_{a}^{*} f=\left(D R_{x}^{*} f\right)(x)\right.$. Thus $D$ is invariant. If $K$ is a field of definition for $G$ over which $s$ and $X_{s}$ are rational, and $x$ is generic for $G$ over $K$, we have $D_{x} f=X_{s} R_{s-1 x}^{*} f$ for $f \in K(G)$ and therefore $D_{x}=X_{s} R_{s-1}^{*}$. By the invariance of $D$ we have $D_{s}=D_{x} R_{x-1 s}^{*}=X_{s}$ $R_{s-1 x}^{*} R_{x-1}^{*}=X_{s}$, and in paticular $\left(D \xi_{i}\right)(s)=X s_{i}$.

Since an invariant $\Omega$-derivation of $\Omega(G)$ is determined by its local component at one point of $G$, the uniqueness is clear.

q.e.d.

In the following we shall denote by $D_{X}$ the element of $\mathfrak{g}$ which is determined by $X$ as described in this lemma.

Let $D \in \mathfrak{g}$, then $d_{1}(x) D$ is in $\mathrm{g}$ for any $x \in G$. Let $V$ be an affine variety in which $x$ has a representative, then

$d_{\imath}(x) D \xi_{i}=L_{x}^{*-1} D L^{*} \xi_{\imath}=L_{x-1}^{*} D \phi^{i}(x, \xi)=L_{x}^{*-1} \sum_{j=1}^{N}\left(\partial \phi^{i}(x, Y) / \partial Y_{j}\right)_{Y^{*}=\xi} \chi_{j}(\xi)$.

And we have that $d \iota(x) D$ is defined over $k(x)$ if $D$ is defined over $k$. Further we have

LEMMN 2. Let $H$ be a connected algebraic subgroup of $G$ with the Lie algebra $\mathfrak{h}$. Then for any $h \in H, d \iota(h)$ maps $\mathfrak{y}$ iuto itself.

Proof. Let $D \in \mathfrak{h}$; let $k$ be a field of definition for $G, H$ and $D$ over which $h$ is rational ; let $m$ be the maximal ideal of the local ring of a generic point over $k$ on $H$. Then for $f \in \mathfrak{m} \cap k(G), L_{h}^{*} f$ is in $\mathfrak{m} \cap k(G)$ and therefore $D L_{h}^{*} f$ is in $\mathfrak{m} \cap k(G)$, since $D \in \mathfrak{h}$. So we have $L_{h-1}^{*} D L_{h}^{*} f \in \mathfrak{m} \cap k(G)$, i.e. $d \iota(x) D$ is in $\mathfrak{h}$.

q.e.d.

Let $D_{1} \ldots \ldots D_{n}$ be a base of $g(, k), k$ being a field of definition for $G$, then for any generic point $x$.over $k$ on $G$ we may express $d \iota(x) D_{i}=\sum_{j=1}^{n} \gamma_{j i}$ $D_{j}$, where $\gamma_{j i}$ is in $\Omega$. Put $D_{i} \xi_{j}=\chi_{i j}(\xi)$ and $d_{l}(x) D_{i} \xi_{j}=\chi_{j}(\xi)$, then $\chi_{j i}(\xi)$ is in $k(\xi)$ and $\chi_{j}(\xi)$ is in $k(x)(\xi)$. Since $D_{1}, \ldots, D_{n}$ is a base of $g$, a family of vectors $\left(\chi_{11}(\xi), \ldots, \chi_{1 N}(\xi)\right), \ldots,\left(\chi_{n_{1}}(\xi), \ldots, \chi_{n}(\xi)\right)$ are linearly independent over $\Omega$. Therefore $\chi_{j}(\xi)=\sum_{l=1}^{n} \gamma_{l i} \chi_{l j}(\xi)$ implies that $\gamma_{i j}$ is an element $\gamma_{i j}(x)$ of $k(x)$. If we denote by $A d(x)$ the matrix $\left(\gamma_{i},(x)\right)$, we have that $x \rightarrow A d(x)$ is a rational mapping $A d$ of $G$ into $g l(n, \Omega)$ defined over $k$. For independent 
generic points $x$ and $y$ over $k$ on $G$, we have $d \iota(x) d \iota(y)=d \iota(x y)$, so $A d(x) A d(y)$ $=A d(x y)$. And $d_{\iota}\left(x^{-1}\right)$ being the inverse of $d \iota(x)$, we have that $A d(x)$ is in $G L(n, \Omega)$. Thus $A d$ is everywhere defined on $G$ and the locus of $A d(x)$ over $k$ on $G L(n, \Omega)$ is a connected algebraic subgroup of $G L(n, \Omega)$ which is denoted by $\operatorname{Ad}(G)$ (cf. proposition 2 of [1] p.82). So $x \rightarrow A d(x)$ is a rational homomorphism of $G$ onto $A d(G)$ defined over $k$.

Now for this rational homomorphism $x \rightarrow A d(x)$ of $G$ onto $A d(G)$, we have the natural homomorphism ad of $\mathfrak{g}$ onto the Lie algebra of $\operatorname{Ad}(G)$. Then we may suppose that $a d(\mathfrak{g})$ is contained in $\mathfrak{g l}(n, \Omega)$ and that $\operatorname{ad}(\mathfrak{g})$ is a subalgebra of the Lie algebra of all endomorphisms of the vector space $\mathfrak{g}$ over $\Omega$.

Let $k^{\prime}$ be another field of definition for $G$ and let $D_{1}^{\prime}, \ldots \ldots D_{n}^{\prime}$ be a base of $\mathfrak{g}\left(, k^{\prime}\right)$. Let $A d^{\prime}$ and $a d^{\prime}$ be the representation of $G$ and its differential which are defined as described above with respect to $k^{\prime}$ and $D_{1}^{\prime}, \ldots . D_{n}^{\prime}$. Then if $K$ is the compasitum of $k$ and $k^{\prime}$, there exists a matrix $S=\left(s_{i j}\right)$ in $G L(n, K)$ such that $D_{i}=\sum_{i=1}^{n} s_{j i} D_{j}^{\prime}$. We have $A d^{\prime}(x)=S A d(x) S^{-1}$ for any $x \in G$. Let $D$ be an element of $\mathfrak{g}$ defined over $K$. Then from the definition and (3) we have that $\Phi(a d(D))=-\left(\Lambda_{i j}\right)$ and $\Phi\left(a d^{\prime}(D)\right)=-\left(\Lambda^{\prime}{ }_{i j}\right)$, where $\Lambda_{1}$, $=\left(D \gamma_{i j}(\xi)\right)(e)$ and $\Lambda_{i j}^{\prime}=\left(D \gamma_{i j}^{\prime}(\xi)\right)(e)$. It is easily seen that $\Phi\left(a d^{\prime}(D)\right)=S$ $\Phi(\operatorname{ad}(D)) S^{-1}$.

Then if we identify $A d(x)$ with $d_{\iota}(x)$ and $a d(x)$ with the endomorphism of the vector space $g$ over $\Omega$ such that $D_{\imath} \rightarrow \sum_{l=1}^{n} \Lambda_{\jmath i} D_{y}$, we have a linear representation of $G$ and its differential which are independent of the choice of a field $k$ of definition for $G$ and a base $D_{1}, \ldots, D_{n}$ of $\mathrm{g}(, k)$. We shall call $A d$ and $a d$ the adjoint representation of $G$ and $\mathfrak{g}$, respectively. Then we have

Proposition 1. Let $G$ be a connected algebraic group; let $x \rightarrow A d(x)$ be the adjoint representation of $G$. Then for any $D, D^{\prime} \in \mathfrak{g}$ we have ad(D) $D^{\prime}=$ $\left[D, D^{\prime}\right]$.

Proof. Let $k$ be a field of definition for $G$ and $D$; let $D_{1}, \ldots, D_{n}$ be a base of $\mathrm{g}(, k)$; let $x$ be a generic point over $k$ on $G$. Then we have

$$
A d(x) D_{i}=\sum_{-1}^{\eta} \gamma_{j i}(x) D_{,}
$$

where $\gamma_{, i}(x)$ is in $k(x)$. Let $\xi_{1}, \ldots \ldots, \xi_{v}$ be coordinate functions of $G$ relative to an affine variety in which the unit element $e$ has a representative, and put $D \xi_{j}=\chi_{1}(\xi), D_{i} \xi_{i}=\chi_{i j}(\xi)$ and

$$
\Lambda_{\imath j}=\left(D \gamma_{i},(\xi)\right)(\boldsymbol{e}) \text {. }
$$

Then from (3) and the above remark it follows that

$$
\operatorname{ad}(D) D_{i}=-\sum_{l=1}^{\imath} \Lambda_{l i} D_{l}
$$

and therefore 


$$
a d(D) D_{\imath} \xi_{j}=-\sum_{=1}^{n} \Lambda_{l 2} \chi_{l i}(\xi)
$$

On the other hand

$$
\left[D, D_{i}\right] \xi_{i}=\sum_{p=1}^{v}\left\{\partial \chi_{i j}(\xi) / \partial \xi_{,} \chi_{1 \prime}(\xi)-\partial \chi_{j}(\xi) / \partial \xi_{1} \chi_{i p}(\xi)\right\} .
$$

So it is sufficient to show that these two functions (7) and (8) have the same value at $\boldsymbol{e}$. From (5) we have

$$
\sum_{x^{\prime}=1}^{n} \gamma_{l l}(x) \chi_{l},(\xi)=L_{x-1}^{*} D_{i} L_{x}^{*} \xi_{j}
$$

Applying $L_{x}^{*}$, we have

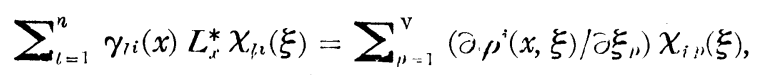

so at $e$, we have

$$
\sum_{l=1}^{n} \gamma_{l i}(x) \chi_{l i}(x)=\sum_{l=1}^{v}\left(\partial \gamma^{j}(x, Y) / \partial Y_{l \prime}\right)_{r=e} \chi_{i \prime \prime}(e)
$$

Applying the $k$-derivation of $k(x)$ induced by $D$, we have

$$
\begin{aligned}
& \sum_{l=1}^{n, N} \gamma_{l i}(x) / \partial x_{l}, \chi_{p}(x) \chi_{l}(x)+\sum_{l=1 p=1}^{n, v} \gamma_{l}(x) \partial \chi_{l j}(x) / \partial x_{l}, \chi_{l}(x) \\
& =\sum_{p, q=1}^{\mathrm{V}}\left(\partial \mathcal{P}^{\jmath}(X, Y) / \partial X_{\imath,} Y_{q}\right)_{Y=x, Y=\rho} \chi_{q}(x) \chi_{\imath p}(e) .
\end{aligned}
$$

Since $\gamma_{i j}(x)$ is in the specialization ring of $e$ in $k^{\prime}(x)$ and $\gamma_{i}(e)=\delta_{i}$, putting $x=e$, we see that the functions (7) and (8) have the same value at $e$ (cf. (2) and (6)).

q.e.d.

2. In this section we assume that the characterestic of the universal domain is 0 . We first prove the proposition which affords the definition of replica.

Proposition 2. Let $H_{1}$ aud $H_{2}$ be connected algebraic subgroups of $\mathrm{G}$ whose Lie algebras are $\mathrm{y}_{1}$ and $\mathrm{y}_{2}$; let $H_{0}$ be the component of $H_{1} \cap H_{2}$ containing the unit element $e$. Then we have $\mathfrak{h}_{1} \cap \mathfrak{h}_{2}=\mathfrak{h}_{0}$ where $\mathfrak{h}_{0}$ is the Lie algebra of $H_{0}$.

Proof. $\quad H_{0}$ being an algebraic subgroup of $H_{1}$ and $H_{2}$, clearly $\mathfrak{h}_{0} \subset \mathfrak{h}_{1} \cap \mathfrak{h}_{2}$.

Let $D$ be any element of $\mathfrak{h}_{1} \cap \mathfrak{h}_{2}$. We shall show that $D$ is in $\mathfrak{y}_{0}$. Let $k$ be a field of definition for $G, H_{0}, H_{1}, H_{2}$ and $D$; let $x$ be a generic point over $k$ on $G$; let $V$ be an affine variety in which $e$ has a representative, then $H_{i}$ also has a representative in $V$. Let $\mathfrak{B}$ be the ideal in $k[X]$ determined by $V$; let $\Re_{i}$ be those for $H_{i}$, then the set of those points of $G$ whose represen'atives in $V$ are zeros of any polynomials in $\mathfrak{A}=\mathfrak{F}_{1}+\mathfrak{F}_{2}$ is the set of those points of $H_{1} \cap H_{2}$ which have representatives in $V$. Let $\Sigma_{0}$ be the set of all those $P(X)$ in $k[X]$, for which there exists a polynomial $L(X)$ in $k[X]$ such that $L\left(h_{0}\right) \neq 0$ and $L(X) P(X) \in \mathfrak{P}$, where $h_{0}$ is a generic point over $k$ on $H_{0}$. Then by the lemma 5 of [5] F-III ${ }_{3}, Q_{0}$ is a $\Re_{0}$-primary ideal in $k[X]$. Let $h_{i}$ be a generic point over $k$ on $H_{i}$; let $Q_{i}$ be the specialization ring of $h_{i}$ in $k(x)$; let $\mathfrak{m}_{i}$ be 
the maximal ideal in $Q_{\imath}$. Then we have $\mathfrak{m}_{\imath}=\mathscr{W}_{i \mid r} Q_{\imath}$, where $\mathfrak{F}_{i \mid x}$ is the set of those elements $P(x)$ of $k[x]$ for some $P(X) \in \mathcal{P}_{\text {. }}$. We shall denote by the same $D$ the $k$-derivation of $k(x)$ induced by $D$. Then, since $D$ is everywhere finite and in particular $D Q_{0} \subset Q_{0}$, for $D$ to be contained in $\mathfrak{h}_{k}$, it is sufficent that $D \mathbb{H}_{0 \mid x} \subset \mathbb{M}_{0}$. Take any element $P(x)$ of $\mathfrak{P}_{0 \mid x}$, then $D P(x) \in Q_{0}$, so we may express $D P(x)=F_{1}(x) / F_{2}(x)$ for some $F_{1}(X), F_{2}(X) \in k[X]$ such that $\mathrm{F}_{2}\left(h_{0}\right) \neq 0$. Let $P(X)$ be an element of $k[X]$ which has a specialization $P(x)$ over $(X) \rightarrow(x)$. Let $r$ be the minimal positive integer such that $P(X)^{r} \in \mathfrak{D}_{0}$, then there exists $L(X) \in k\lceil X]$ such that $L\left(h_{0}\right) \neq 0$ and $P(X)^{r} L(X) \in \mathfrak{R}$, and we may write

$$
P^{\prime}(X)^{r} L(X)=P_{1}(X)+P_{2}(X),
$$

where some $P_{\imath}(X) \in \mathcal{H}_{1}$, therefore we have

$$
P(x)^{r} L(x)=P_{1}(x)+P_{2}(x) .
$$

Applying $D$ on this relation, we have

$$
\begin{aligned}
& \left.r P(x)^{r-1} D P^{\prime} x\right) L(x)+P(x)^{r} D L(x)=D P_{1}(x)+D P_{2}(x), \text { i. e. } \\
& r P(x)^{r-1} L(x) F_{1}(x) / F_{2}(x)=D P_{1}(x)+D P_{2}(x)-P(x)^{r} D L(x) .
\end{aligned}
$$

Since $D$ is in $\mathfrak{h}_{1} \cap \mathfrak{h}_{2}, D P_{i}(x)$ is in $\mathrm{nl}_{i}(i=1,2)$, and therefore we may write $D P_{\imath}(x)=P_{i}^{\prime}(x) / P_{i}^{\prime \prime}(x)$ for some $P_{i}^{\prime}(X) \in \mathfrak{P}_{\imath}, P_{i}^{\prime \prime}(X) \in k[X]$ such that $P_{\imath}^{\prime \prime}\left(h_{0}\right) \neq 0$, since $D P_{i}(x)$ is in $Q_{0}$. So, $D L(x)$ being in $Q_{0}$, in the relation

$$
r P(x)^{r-1} L^{2}(x) F_{1}(x) / F_{2}(x)=L(x)\left\{D P_{1}(x)+D P_{2}(x)\right\}-L(x) P(x)^{r} D L(x),
$$

the right hand side may be expressed as $A(x) / B(x)$ for some $A(X) \in \mathfrak{A}$ and $B(X) \in k[X]$ such $B\left(h_{v}\right) \neq 0$. Then we have

$$
r P(x)^{r-1} L^{2}(x) B(x) F_{1}(x)=A(x) F_{2}(x)
$$

and, $x$ being generic for $G$ over $k$, we have

$$
r P(X)^{r-1} L^{2}(X) B(X) F_{1}(X)=A(X) F_{2}(X)+P_{0}(X),
$$

where $P_{0}(X)$ is some element of $\mathscr{B}$. Since $\mathfrak{P}$ is contained in $\mathcal{P}_{i}$, the right hand side is contained in $\mathfrak{A}$, and therefore $r P(X)^{r-1} F_{1}(X)$ is in $\mathfrak{Q}_{0}$. But $r P(X)^{r-1} \notin$ $\mathfrak{Q}_{0}$, so we have that $F_{1}(X)$ is in $\mathfrak{X}_{0}$. Thus we have shown that $D$ is in $\mathfrak{h}_{0}$.

q.e.d.

Now we have two corollaries; and the first as follows;

COROLlary 1. Let be a connected algebraic gropu For any element $D$ of the Lie algebra of $G$, there exists the smallest connected algebraic subgroup of $G$ whose Lie algebra contains $D$.

Proof. Let $\mathfrak{M l}$ be the family of connected algebraic subgroups of $G$ whose Lie algebra contains $D$; then, as $\mathfrak{M}$ is not empty, there exists an element $H$ of $\mathfrak{M}$ whose dimension is $>0$ and the minimal in $\mathfrak{M}$. Take any $H^{\prime} \in \mathfrak{M}$, and let $\left(H \cap H^{\prime}\right)_{0}$ be the component of $H \cap H^{\prime}$ containing $e$, then $\left(H \cap H^{\prime}\right)_{0} \subseteq H_{0}$ By the proposition 2, the Lie algebra of $\left(H \cap H^{\prime}\right)_{0}$ contains $D$, so $\left(H \cap H^{\prime}\right)_{\mathrm{c}}$ $\in \mathfrak{M}$ and $\operatorname{dim}\left(H \cap H^{\prime}\right)_{0} \geqq \operatorname{dim} H$. Thus we have that $\left(H \cap H^{\prime}\right)=H$ and $H$ is contained in $H^{\prime}$.

q.e.d. 
Let us denote by $G_{D}$ the smallest connected algebraic subgroup $H$ of $G$ in the corollary and by $\mathrm{g}_{D}$ the Lie algebra of $G_{D}$. We call any element of $\mathfrak{g}_{D}$ replica of $D$. This is an extension of the definition 2 of [1] p. 180.

Let $H$ be a connected algebraic subgroup of $G$ with Lie algebra $\mathfrak{n}$; let $\mathrm{H}^{\prime}$ be the connected algebraic subgroup of $G$ generated by $G_{D}$ for all $D \in \mathfrak{l}$; let $\mathfrak{h}^{\prime}$ be the Lie algebra of $H^{\prime}$. Then $H^{\prime}$ is contained in $H$, since each $G_{D}$ is contained in $H$. But, as $\mathfrak{l}$ is generated by $\mathfrak{g}_{D}$ for all $D \in \mathfrak{h}$, $\mathfrak{l}^{\prime}$ contains $\mathfrak{h}$, therefore $\operatorname{dim} H=\operatorname{dim} \mathfrak{h} \leqq \operatorname{dim} \mathfrak{y}^{\prime}=\operatorname{dim} H^{\prime}$, and $H=H^{\prime}$. Thus we have proved that $H$ is the connected algabraic subgroup of $G$ generated by $G_{\prime \prime}$, for all $D \in \mathfrak{h}$, and we have another corollary of the proposition 2 :

Corollary 2. Let $H_{1}$ and $H_{2}$ be connocted algebraic subgroups of $G$, let $\mathfrak{l}_{1}$ and $\mathfrak{l}_{2}$ be the Lie algebras of $H_{1}$ and $H_{2}$, respectively. Then if $b_{1}$ contains b), $H_{1}$ contains $H$.

The next proposition shows the relation between $G_{D}$ and rational homomorphism :

Proposition 3. Let $G$ and $G$ be conneted algebraic groups; let $\rho$ be a rational homomorphism of $G$ onto $\bar{G}$. Then for any $D$ of in, we have $\rho\left(G_{n}\right)=$ $G_{a, D}$.

Proof. As $d \rho D$ is in the Lie algebra $d \rho \mathrm{g}_{D}$ of the algebraic subgroup $\rho\left(G_{D}\right)$ of $G, G_{i \rho D}$ is contained in $\rho\left(G_{D}\right)$.

We shall show that $G_{(\alpha)}$ contains $\rho\left(G_{D}\right)$. Let $H$ be the algebraic subgroup of $G$ consisting of those $z$ such that $\rho(z)$ is in $G_{1 \rho D}$ and $H_{0}$ be the component of $H$ containing the unit element $e$ of $G$. Let $k$ be a field of definition for $\rho, D$, and all these algebraic groups concerned; let $x$ and $h$ be generic points over $k$ on $G$ and $H_{0}$, then $y=\rho(x)$ and $\rho(h)$ are those on $G$ and $G_{d \rho p}$. Let $V$ and $V$ be affine varieties in which the unit elements of $G$ and $G$ have their representatives. We shall denote by the same $\rho$ the rational mapping of $V$ into $\bar{V}$ induced by $\rho$. Let $\mathfrak{R}^{\prime}$ and $\mathfrak{B}_{0}$ be the ideals in $k[X]$ determined by $x$ and $h$; let $\mathfrak{B}_{0}$ be the ideal in $k[Y]$ determined by $\rho(h)$. Let $[$ be the subset of $k[X]$ consis ing of those $P(X)$ for which there exists $P_{0}(X) \in k[X]$ such that $P_{n}(x)$ $\neq 0$ and $P(x) / P_{0}(x) \in \bar{N}_{0 \mid \eta}$, where $\bar{F}_{\mid y}$ is the ideal in $k[y]$ consisting of those $F(y)$ for some $F(Y) \in \mathfrak{B}_{0}$; let $\mathfrak{T}$ be the ideal in $k[X]$ generated by $\mathbb{R}$ and $(\mathbb{E}$.

Then for a point $z$ of $G$ which has a representative in $V, z$ is in $H$ if and o.ly if $z$ is a zero of $\mathscr{H}$. In fact; suppose that $z$ is in $H$, then $P(z)=0$ for $P \in \mathcal{B}$. If $P \in \mathbb{E}$, we have $R(x)=P(x) / P_{0}(x) \in k(x)$ such that $R(x) \in \overline{\mathcal{B}_{0 / \gamma}}$. Since $\rho$ is everywhere defined on $G$ and therefore $R(x)$ is in the specialization ring of $z$ in $k(x)$, we have another expression $R(x)=P^{\prime}(x) / P_{0}^{\prime}(x)$ where $P_{1}^{\prime}(z) \neq 0$, if necessary. Since $\rho(z)$ is a specialization of $\rho(h)$ over $k$, we have $P^{\prime}(z)=0$ and therefore $P(z)=0$. Conversely, suppose that $z$ is a zero of $\mathfrak{A}$. For $F \in \mathbb{P}_{0}$ there exist $P(X)$ and $P_{0}(X)$ in $k[X]$ such that $F(y)=P(x) / P_{v}(x)$ and $P_{0}(z) \neq 0$, 
since $F(y)$ is in the specialization ring of $z$ in $k(x)$. Then $z$ being a specialization of $x$ over $k$, we have $P(X) \in \mathbb{E}$, and $P(z)=0$. Thus $F(\rho(z))=0$ and $\rho(z)$ is a specialization of $\rho(\boldsymbol{h})$ over $k$.

Let $D$ be the ideal in $k[X]$ consisting of those $P(X)$ for which there exists $L(X) \in k[X]$ such that $L(h) \neq 0$ and $L(X) P(X) \in \mathfrak{A}$, then $\mathfrak{D}$ is $\mathfrak{F}_{0}$-primary. Let $\bar{m}$ be the maximal ideal of the specialization ring of $\rho(h)$ in $k(y)$, We shall denote by the same $D$ and $d \rho D$ the $k$-derivations of $k(x)$ and $k(y)$ induced by $D$ and $d \rho D$, respectively. Then, from the definition of $d \rho$, we have $D \mathrm{~m}=$ $d \rho D \bar{m} \subset \bar{m}$, since $d \rho D$ is in the Lie algebra of $G_{a \rho p}$. Now if $P(X) \in \mathfrak{P}$, we have $D \cdot P(x)=0$ and if $P(X) \in\left(E\right.$, we have $F(Y) \in \widetilde{\mathcal{F}_{0}}$ and $P_{0}(X) \in k[X]$ such that $P_{0}(x) \neq 0$ and $F(y)=P(x) / P_{0}(x)$. So we have

$$
D P(x)=\left(D P_{\mathrm{v}}(x)\right) F(y)+P_{0}(x) D F(y) \text {. }
$$

Since $D$ is finite at $h, D P_{0}(x)$ is in the specialization ring of $h$ in $k(x)$. On the other hand we have $D F(y)=D F(\rho(x))=d \rho D F(y)=F_{1}(y) / F_{2}(y)$ tor some $F_{1}(Y) \in \overline{\mathcal{F}_{0}}$ and $F_{2}(Y) \in k[Y]$ such that $F_{2}(\rho(h)) \neq 0$. And $\rho$ being defined at $h$, $F(y)$ and $F_{1}(y)$ is in the specialization ring of $h$ in $k(x)$. Thus we may express $D P(x)=A(x) / B(x)$ for some $A(X) \in \mathfrak{A}$ and $B(X) \in k[X]$ such tha: $B(h) \neq 0 . \quad D$ being finite at $h$, we see that this is true for any $P(x) \in \mathfrak{A}$. So the argument which has run in the proof of the proposition 2 shows that $D$ is in the Lie algebra of $H_{0}$. Thus $G_{D}$ is in $H$, and $\rho\left(G_{D}\right)$ is contained in $G_{\| \rho D}$.

q.e.d.

We have an application of this proposition as follows;

Proposition 4. Let $\rho$ be a rational homomorphism of a connected algebraic group $G$ onto another $\bar{G}$; let $H$ be an algebraic subgroup of $\bar{G}$ with the Lie algebra $\mathfrak{h}$. Then the set of those elements $y$ of $G$ such ihat $\rho(y)$ is in $\bar{H}$ is an algebraic subgroup $H$ of $G$ with the Lie algebra consisting of those elements $D$ of $\mathfrak{g}$ such that $d \rho D$ is in $\mathfrak{h}$.

Proof. It is known that such a set $H$ is algebraic. We may suppose that $H$ and $\bar{H}$ are connected. Let $\mathfrak{h}^{\prime}$ be the set of those $D$ of $\mathfrak{g}$ such that $d \rho D$ $\in \overline{\mathfrak{y}}$; let $\mathfrak{h}$ be the Lie algebra of $H$. Since $\rho(H)=H$, we have $d \rho \mathfrak{y}=\mathfrak{h}$ and therefore $\mathfrak{h}$ is contained in $\mathfrak{h}^{\prime}$. Conversely for any $D$ of $\mathfrak{h}^{\prime}$ we have $\rho\left(G_{D}\right)=$ $G_{\text {तlp } D}$ by the proposition 3. As $\bar{H}$ is algebraic, $\bar{H}$ contains $G_{(l \rho D}$. Thus we have that $G_{D}$ is contained in $H$ and $D$ is in $\mathfrak{h}$.

q.e d.

From this proposition follows the next corollary;

COROLLARY. Let $G$ be a connected algebraic group with the Lie algebra $g$; let $\Xi$ be a subspace of $\mathrm{g}$. Then the set $H$ of those elements $y$ of $G$ such that Ad(y) maps $g$ into itself is an algebraic subgroup of $G$ with the Lie algebra consitsing of those elements $D$ of g such that $\left[D, D^{\prime}\right]$ is in for any $D^{\prime} \in$ ?. 
Proof. Let $H$ be the set of $A d(y)$ such that $A d(y) \subset \bar{y}$. Then it is known that $H$ is algebraic, and therefore that $H$ is algebraic. We may suppose that $\bar{H}$ is contained in $G L(n, \Omega)$ where $n$ is the dimension of $G$. By the example of $\$ 10$ of [1], the Lie algebra $\overline{\mathfrak{h}}$ of $\bar{H}$ is the set of those $X$ of $\mathfrak{g l}(n$, $\Omega)$ such that $X \mathfrak{g} \subset$. By the proposition 4 the Lie algebra $\mathfrak{h}$ of $H$ is the set of those $D$ of $\mathfrak{g}$ such that $\operatorname{ad}(D) \in \overline{\mathfrak{h}}$. So, for $D$ of $\mathfrak{g}, D$ is in. $\mathfrak{h}$ if and only if ad $(D) \mathfrak{z} \subset \mathfrak{s}$ i. e. $[D, \mathfrak{g}] \subset \mathfrak{g}(\mathrm{cf}$. Proposition 1).

q.e.d.

3. In this section we assume that the characterestic of the universal domain is 0 . Then we have

Proposition 5. Let $U$ be a subvarieiy of a connected algebraic group $G$ which contains the unit element $e$ : let $k$ be a field of definition for $G$ and $U$. Then the Lie algebra of the connected algebraic subgroup $H$ of $G$ generated by $U$ is the minimal subspace $\mathfrak{h}$ of $\mathrm{g}$ such that (i) for any $y \in U, A d(y)$ maps $\mathfrak{h}$ into itself, (ii) for any overfield $k^{\prime}$ of $k$, generic point $u$ over $k^{\prime}$ on $U$ and $k^{\prime}$-derivation $X$ of $k^{\prime}(u), D_{X}$ is in $\mathfrak{h}$.

Proof. Evidently the intersection of those subspaces of $\mathfrak{g}$ with these properties also has these properties too, so there exists the unique minimal subspace $\mathfrak{y}$ of $\mathfrak{g}$. By the lemma 1 and 2 the Lie algebra of $H$ satisfies these two conditions, and therefore $\mathfrak{h}$ is contained in the Lie algebra of $H$.

We shall show the converse. Let $D$ be an element of the Lie algebra of $H$, defined over $k$; let $u_{1}, \ldots, u_{r}$ be independent generic points over $k$ on $U$ such that product $h=u_{1} \ldots . u$. is a generic point over $k$ on $H$; let $\xi_{1}, \ldots$, $\xi_{N}$ be coordinate functions of $G$ relative to an affine variety $V$ in which $e$ has a representative; let $X$ be the $k$-derivation of $k(h)$ induced by $D$ : put $K=k\left(u_{1}, \ldots, u_{r}\right)$ and $K_{i}=k\left(u_{1}, \ldots, \hat{u}_{i}, \ldots, u_{i}\right)$, where $\wedge$ means that the letter under $\wedge$ is to be omitted. Then there exists a $k$-derivation of $K$ which is an extension of $X$, and which we shall denote by the same $X$. Let $X_{i}$ be the $K_{\imath}$-derivation of $K$ such that $X_{i} u_{i j}=X u_{i j}$, where $u_{i j}$ is the $j$-th coordinate of the representative of $u$, in $V(1 \leqq i \leqq r, 1 \leqq j \leqq N)$. In fact there exists such $X_{i}$, since $K_{i}$ and $k\left(u_{1}\right)$ are linearly disjoint over $k$. Then we have $X=$ $\sum_{i=1}^{r} X_{i}$. Put $s_{1}=e, s_{i}=\Pi_{p<\iota} u_{l}, t_{i}=\Pi_{\iota<1} u_{g}$ and $t_{r}=e(1 \leqq i \leqq r)$, then we have

$$
D=\sum_{i=1}^{r} A d\left(s_{i}\right) D_{X_{i}} .
$$

In fact; it is sufficent to prove that the eqaulity of these two invariant derivations holds at $h$, i. e. putting $D \xi_{i}=\chi_{i}(\xi)$ and $A d\left(s_{i}\right) D_{x_{i}} \cdot \xi_{j}=\chi_{i j}(\xi)$, we have to show that $\chi_{j}(h)=\sum_{i=1}^{r} \chi_{i,}(h)$. We have

$$
\chi_{i}(h)=X h_{\jmath}=\sum_{i=1}^{\prime \prime} X_{i}\left(s_{i} u_{i} t_{i}\right)_{j}
$$




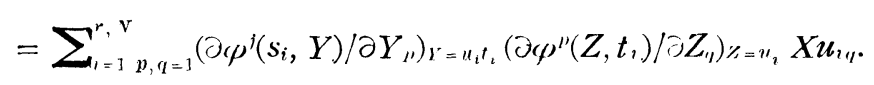

On the other hand we have

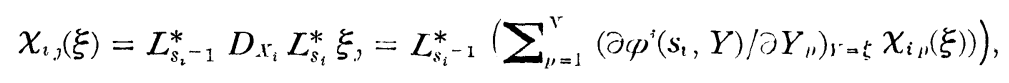

where $\bar{\chi}_{i,}(\xi)=D_{x_{i}} \xi_{l}$. So, by the invariance of $D_{r_{i}}$

$$
\begin{aligned}
& \chi_{\iota,}(h)=\sum_{p=1}^{\mathrm{v}}\left(\partial \phi^{i}\left(s_{i}, Y\right) / \partial Y_{\mu}\right)_{Y=u_{\imath} \iota_{i}} \chi_{i \mu}\left(u_{i} t_{i}\right) \\
& =\sum_{p, q=1}^{r}\left(\partial \varphi^{*}\left(s_{i}, Y\right) / \partial Y_{\mu^{\prime}}\right)_{Y=u_{i} t_{i}}\left(\partial \varphi^{\prime \prime}\left(Z, t_{i}\right) / \partial Z_{q}\right)_{\eta=u_{i}} \chi_{i q}\left(u_{i}\right) .
\end{aligned}
$$

By the lemma $1, \bar{\chi}_{i_{q}}\left(u_{\imath}\right)=X_{\imath} u_{i_{q}}=X u_{i q}$. Thus we have proved (9).

For $y_{1}, y_{2} \in U$, we have $A d\left(y_{1} y_{2}\right)=A d\left(y_{1}\right) A d\left(y_{2}\right)$ and therefore $A d\left(y, y_{2}\right)$ h $\subset \mathfrak{l}$. Thus, from (i) and (ii) it follows that $D$ is in $\mathfrak{h}$.

q. e.d.

Now we prove the main theorem;

TheOREM. Let $G$ be a connected algebraic group with the Lie algebra is: let $H_{i}$ be connected algebraic subgroups of $G$ with the Lie algebras $\mathfrak{h}_{i}$, where $i$ runs through a set $I$ of indecies; let $\mathfrak{h}$ be the subalgebra of a generated by all $\mathfrak{h}_{i}, s$; let $H$ be the connected algebraic subgroup of $G$ generated by all $H ; s$. Then the Lie algebra of $H$ is $\mathfrak{h}$.

Proof. Suppose that $I$ is a finite set, say $I=\{1, \ldots, r\}$. Lat $k$ be a field of definition for $H_{1}, \ldots, H$, and $G$; let $h_{1}, \ldots, h_{r}$ be independent generic points over $k$ on $H_{1}, \ldots, H_{r}$, respectively ; let $U$ be the locus of the product $u=h_{1} \ldots h_{r}$ over $k$ on $G$, then $H$ is the connected algebraic subgroup of $G$ generated by $U$. Let $\mathfrak{h}^{\prime}$ be the Lie algebra of $H$. Since $H$ contains $H_{i}$, so $\mathfrak{h}^{\prime}$ contains $\mathfrak{h}_{i}$ and therefore $\mathfrak{l}^{\prime}$ contains $\mathfrak{h}$.

To prove the converse we have to show that $y$ satisfies the two conditions of the proposition 5 .

As for (i); Let $\bar{H}$ be the set of those elements $x$ of $G$ such that $A d(x)$ maps $\mathfrak{h}$ into itself, then $H$ is an algebraic group (cf. the corollary of the proposition 4). Let $H_{0}$ be the component of $H$ containing $e$, then, by the same corollary the Lie algebra $\overline{h_{0}}$ of $\overline{H_{0}}$ is the subalgebra of $\mathfrak{g}$ consisting of those $D$ of gsuch that $[D, \mathfrak{b}] \subset \mathfrak{y}$, so all $\mathfrak{h}$ s $s$ are in $\overline{\mathfrak{h}}$, and by the corollary 2 of the proposition 2, all $H_{i} s$ are in $\bar{H}_{0}$ and therefore $H$ is contained in $\bar{H}_{0}$. Thus we proved that the condition (i) is satisfied.

As for (ii); let $k^{\prime}$ be an overfield of $k$; let $u$ be a generic point over $k$ on $U$; let $X$ be any $k^{\prime}$-derivation of $k^{\prime}(u)$. Let $h_{1}^{\prime}, \ldots, h_{r}^{\prime}$ be independent generic points over $k^{\prime}$ on $H_{1}, \ldots, H_{r}$, respectively, then $u^{\prime}=h_{1}^{\prime} \ldots h_{r}^{\prime}$ is a generic point over $k^{\prime}$ on $U$. So we have a $k^{\prime}$-isomorphism of $k^{\prime}\left(u^{\prime}\right)$ which transforms $u$ into $u^{\prime}$. Let $X^{\prime}$ be the $k^{\prime}$-derivation of $k^{\prime}\left(u^{\prime}\right)$ induced by the $X$. As in the proof of the proposition 5 we extend $X^{\prime}$ into $K$ and define the $K_{\imath}$ - 
derivation $X_{i}^{\prime}$ of $K$, where $K=k^{\prime}\left(h_{1}^{\prime} \ldots, h_{r}^{\prime}\right)$ and $K_{i}=k^{\prime}\left(h_{1}^{\prime}, \ldots, \hat{h_{i}^{\prime}}, \ldots h_{r}^{\prime}\right)$, and we have $D_{X^{\prime}}=\sum_{\imath=1}^{r} A d\left(s_{i}\right) \quad D_{X_{i}^{\prime}}$, where $s_{i}$ is a product of some finite points of $U$. From the definition $D_{X^{\prime}}$ is in $\mathfrak{h}_{i}$, and now that the condition (i) has been proved, we have $D_{X^{\prime}} \in \mathfrak{h}$. But from the definition the local components of $D_{X}$ and $D_{x^{\prime}}$ at $u^{\prime}$ are same, and therefore we have $D_{x}=D_{x^{\prime}}$. Thus the condition (ii) has been proved.

In the infinite case, for any finite subset $E$ of $I$, let $\mathfrak{h}_{E}$ be the subalgebra of $g$ generated by all $\mathfrak{h}_{i}$ for $i \in E$; let $H_{E}$ be the connected algebraic subgroup of $G$ with the Lie algebra $\mathfrak{h}_{E}$; let $\mathfrak{h}_{E_{0}}$ be such one of those $\mathfrak{h}_{E}$ that $\operatorname{dim} \mathfrak{h}_{E_{0}}$ is the maximal. Then if $E_{0}$ is a subset of $E$, have $\mathfrak{h}_{F_{0}}=\mathfrak{h}_{k}$. So we have $\mathfrak{h}=\mathfrak{h}_{E_{0}}$.

q.e.d.

The next corollary gives a characterization of algebraic subalgebra of $\mathfrak{b}$, which is a generalization of the proposition 2 of [1] p. 181.

COROLLARY. Let $\mathrm{g}$ be the Lie algebra of a connscted algebraic group; let $\mathfrak{h}$ be a subalgebra of $\mathfrak{g}$. Then $\mathfrak{h}$ is algebraic if and only if for $D \in \mathfrak{h}$ any replica of $D$ is in $\mathfrak{h}$.

Proof. The necessity is trivial from the definition of replica. Conversely, $\mathfrak{h}$ being generated by $\mathfrak{h}_{D} s$ for $D \in \mathfrak{h}, \mathfrak{h}$ is algebraic (cf. the theorem).

q.e.d.

\section{REFERENCES}

[1] C. Chevaliey, Theorie des groupes de Lie II. Actualités Sci. et Industr.1152, Hermann, Paris. 1951.

[2] S. NAKANO, On inivarant differential forms on group varieties. Journ. Math. Soc. Japan, 2 (1950-51), 216-227.

[3] M. Rosent.ICHT, A note of derivations and differentials on algebraic varieties. Portugaliae Math. 16(1957), 44-55.

[4] M. RosENI.ICHT, Some basic theorems of algebraic groups, Amer. Journ. Math. 78(1956), 401-443.

[5] A. WEIL, Founations of algebraic geometry. Amer. Math. Soc. Colloqu um Pub. New York 1946.

MATHEMATICAL INSTITUTE, TÔHOKU UNIVERSITY. 